\title{
EGFR Antibody Conjugated Bimetallic Au@Ag Nanorods for Enhanced SERS-Based Tumor Boundary Identification, Targeted Photoacoustic Imaging and Photothermal Therapy
}

\author{
Shouhui Chen ${ }^{1,2}$, Chenchen Bao ${ }^{1}$, Chunlei Zhang ${ }^{1}$, Yao Yang ${ }^{1}$, Kan Wang ${ }^{1}$ \\ Bhaskara V. Chikkaveeraiah ${ }^{2,3}$, Zhihua Wang ${ }^{1,2}$, Xinglu Huang ${ }^{2}$ \\ Fei Pan $^{1}$, Kun Wang ${ }^{1}$, Xiao Zhi ${ }^{1}$, Jian Ni ${ }^{1}$, Jesus M. de la Fuente ${ }^{1,5}$, Jie Tian ${ }^{4}$ \\ ${ }^{1}$ Institute of Nano Biomedicine and Engineering, Key Laboratory for Thin Film and Microfabrication of Ministry of Education, \\ Department of Instrument Science and Engineering, Shanghai Engineering Center for Intelligent Diagnosis and Treatment Instrument, \\ National Center for Translational Medicine, Collaborative Innovational Center for System Biology, Shanghai Jiao Tong University, \\ Shanghai 200240, China. \\ ${ }^{2}$ Laboratory of Molecular Imaging and Nanomedicine (LOMIN), National Institute of Biomedical Imaging and Bioengineering \\ (NIBIB), National Institutes of Health (NIH), Bethesda, Maryland 20892, USA. \\ ${ }^{3}$ Microfabrication and Microfluidics Unit, Biomedical Engineering and Physical Science Shared Resource, National Institutes of \\ Health (NIH), Bethesda, Maryland 20892, USA. \\ ${ }^{4}$ Intelligent Medical Research Center, Institute of Automation, Chinese Academy of Sciences, Beijing 100190, China. \\ ${ }^{5}$ Instituto de Nanociencia de Aragon (INA), Universidad de Zaragoza, Zaragoza, 50018, Spain.
}

Corresponding authors. E-mail: jiannihome@yahoo.com; jesusmartinezdelafuente@gmail.com

Received: Dec. 22, 2016; Accepted: Dec. 27, 2016; Published: Dec. 30, 2016.

Citation: Shouhui Chen, Chenchen Bao, Chunlei Zhang, Yao Yang, Kan Wang, Bhaskara V. Chikkaveeraiah, Zhihua Wang, Xinglu Huang, Fei Pan, Kun Wang, Xiao Zhi, Jian Ni, Jesus M. de la Fuente, and Jie Tian, EGFR Antibody Conjugated Bimetallic Au@Ag Nanorods for Enhanced SERSBased Tumor Boundary Identification, Targeted Photoacoustic Imaging and Photothermal Therapy. Nano Biomed. Eng., 2016, 8(4): 315-328. DOI: 10.5101/nbe.v8i4.p315-328.

\begin{abstract}
The development of multifunctional nanoprobes for simultaneous targeted imaging, tumor boundary identification and photothermal therapy of gastric cancer has become a great challenge. Herein, EGFR monoclonal antibody-conjugated Au@Ag nanorods decorated with DTNB nanoprobes (5,5'-Dithiobis-(2-nitrobenzoic acid)) (EGFR-Au@Ag NR nanotags) were prepared and characterized. Their biocompatibility was analyzed by MTT assay. In vitro studies show that EGFR-Au@Ag NR nanotags own good biocompatibility and capability of targeting and entering into gastric cancer MGC803 cells, silver nano-film layer on the surface of gold nanorods remarkably enhance surfaceenhanced rama spectra (SERS) signal, enhanced photoacoustic imaging efficacy and photothermal conversion efficiency of gold nanorods. In vivo studies show that prepared nanotags could target actively gastric cancer cells at $2 \mathrm{~h}$ post-injection, and distributed in the tumor site, exhibited enhanced SERS signals to display clearly tumor boundary, enhanced photoacoustic imaging to display clearly tumor boundary. Under $1 \mathrm{w} / \mathrm{cm}^{2}$ laser irradiation, tumor growth was remarkably inhibited by local photothermal therapy. In conclusion, high performance EGFR-antibody conjugated Au@Ag nanorodDTNB nanoprobes exhibit great potential in applications such as targeted photoacoustic imaging, simultaneous tumor boundary identification and selective photothermal therapy of gastric cancer in the near future.
\end{abstract}

Keywords: Gastric cancer; Au@Ag nanorods; SERS imaging; Photoacoustic imaging; Photothermal therapy; Tumor boundary 


\section{Introduction}

Gastric cancer is the fourth commonest tumor and the second leading cause of cancer-related death in the world [1, 2]. In china, gastric carcinoma incidence ranks no. 2 among all tumors; its mortality rate is no. 3 [3]. The current prognosis of gastric cancer is very poor with 5-year survivals of less than 24\% [4]. How to reduce its mortality rate and extend gastric cancer patent life span is still a challengeable problem. Up to date, surgery, radiation and chemotherapies are still effective choice for early and in situ gastric cancers [57], but how to realize precise location of gastric cancer boundary is still a problem. Although in the course of operation, freeze-tissue section and pathological staining observation are still very effective method, but operation needs stopping and wait for the pathological results for one or two hours. Therefore, how to recognize quickly the boundary of gastric cancer in vivo is still a key technological challenge for surgery doctors.

As precision medicine was put forward, multifunctional nanoprobes-based multimode molecular imaging exhibits great potential in applications such as precision theranostics of tumor. In our previous work, with the aim of finding early gastric cancer, we screen out some new biomarkers associated with early gastric cancer and develop novel ultrasensitive detection methods [8-9]. We also designed and fabricated some multifunctional nanoprobes for targeted imaging and simultaneous therapy of subcutaneous and in situ gastric cancer tissues with $5 \mathrm{~mm}$ in diameter [1022]. Among those multifunctional nanoprobes, RGDconjugated gold nanorods could target tumor tissues and kill tumor cells via photothermal therapy [14], exhibiting potential clinical application prospects. How to take advantages of gold nanorods to realize targeted imaging, boundary identification and simultaneous therapy of gastric cancer have become our concerns.

Gold nanorods (GNRs) own two surface plasmon absorption bands. One is transverse plasmon band, relating to the absorption and scattering of light along short axis of rods, located in the visible region at ca. $520 \mathrm{~nm}$, and the other is called longitudinal plasmon band, relating to the absorption and scattering of light along long axis of rods, located in the near-infrared region (NIR) of electromagnetic spectrum which is tunable by changing the aspect of GNRs [23-25]. Because biological tissues are relatively transparent in NIR; therefore, gold nanorods-based theranostic agents for multi-modality imaging and photodynamic and photothermal therapy of tumor own obvious advantages over biological tissues, and have been actively investigated their application. However, Au nanords-based photothermal conversion efficiency is still very low. How to enhance photothermal conversion efficiency of gold nanorods has become a key problem for their clinical translation. Therefore, the development of new strategy to enhance photothermal conversion efficiency of gold nanorods is very necessary.

In recent years, gold anoparticles-based surface enhanced Raman spectroscopy (SERS) has gained more and more attention because of its ability for highly sensitive detection of low concentration of analytes through amplification of electromagnetic field signals by the excitation of localized surface plasmon resonance [26]. Up to date, different shapes of $\mathrm{Au}$ nanoparticles such as urchin-like AuNPs [27, 28], nanoshells [29], gold nanoprism [30], nanoclusters [31], and nanocages [32], have been used as enhanced contrast agents for SERS imaging of cancer cells and ultrasensitive detection of biomarkers. These gold nanoparticles surrounded by Raman reporters provide light emissions that are over 200-times brighter than quantum dots when in direct contact with the metal surface. Up to date, Au NPs-based SERS is emerging as a new theranostic platform for tumor boundary identification, exhibiting the following advantages: 1) minimization of photobleaching and peak overlapping; 2) high spectral specificity; 3) low signal-to-noise ratio in complex biological systems; and 4) enhanced SERS signal. Au nanoparticles-based SERS enables the detection of very low concentration of analytes, even single molecule with high sensitivity and specificity [33-35]. Up to date, few report is closely associated with gold nanorod-based SERS for detection and imaging of analytes. The development of Au nanorodsbased multifunctional SERS nanoprobes for gastric cancer boundary identification has become our concern.

In recent years, bimetallic nanomaterials as SERS substrates are gaining more and more attention [36]. $\mathrm{Ag}$ is known to be more effective plasmonic substrate than Au, whereas Au offers more chemical stability and biocompatibility. Bimetallic Au and Ag nanomaterials offer excellent features in terms of chemical and plasmonic properties. Previous studies showed that Au/Ag bimetallic nanoparticles exhibit higher SERS activity than the pure Au or Ag nanoparticles, and can 


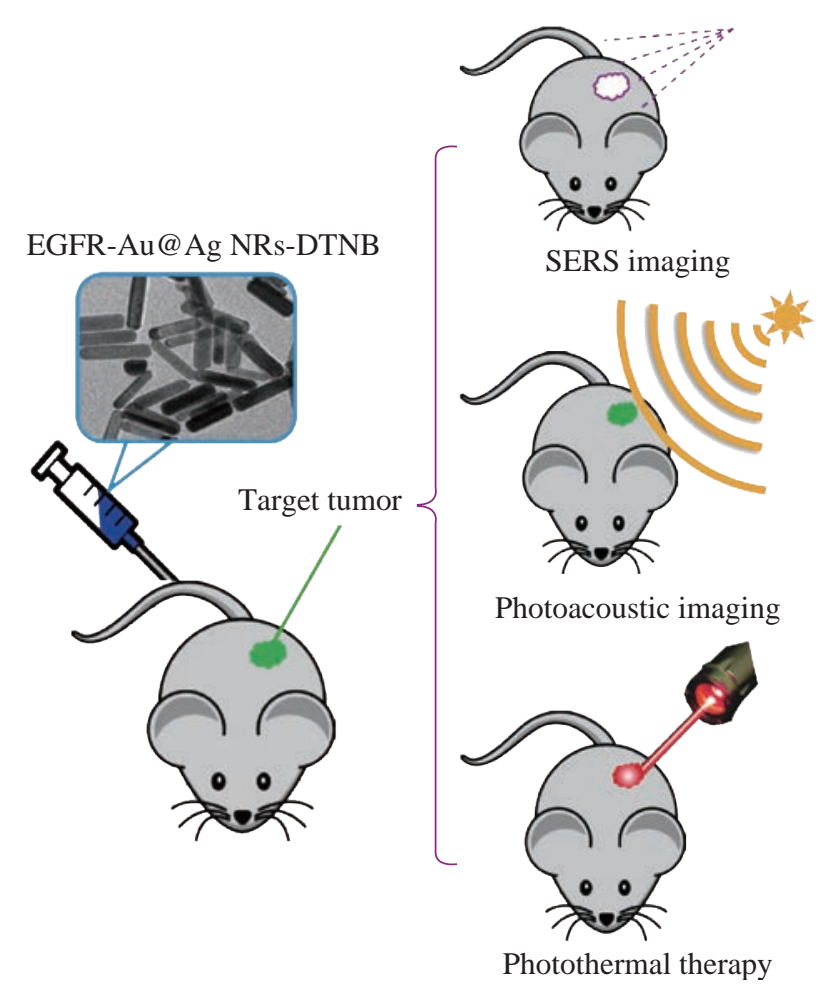

Scheme 1 Schematic of EGFR-Au@Ag NRs nanotags for SERS imaging, photoacoustic imaging and photothermal therapy of gastric cancer-bearing nude muse models.

be used for ultrasensitive detection of biomarkers [3739]. However, up to date, few report is associated with the use of bimetallic Ag@Au nanorods as theranostic agent for in vivo targeted SERS imaging for tumor boundary identification, photoacoustic imaging and simultaneous photothermal therapy.

In this study, thin layer of nanosliver coated gold nanorods (Ag@Au nanorods) were synthesized and characterized, DTNB (5,5'-Dithiobis-(2-nitrobenzoic acid)) was selected as Raman reporter; EGFR overexpressed in the surface of gastric cancer MGC 803 cells was selected as target molecule; EGFR monoclonal antibody conjugated Ag@Au nanorodsDTNB (EGFR-Au@Ag NRs-DTNB ) were prepared, systematic in vitro and in vivo experiments are designed to carefully evaluate the biocompatibility, targeting ability of gastric cancer cells, enhanced SERS signals for boundary identification, photoacoustic imaging and photothermal therapy efficacy of gastric cancer (as shown in Scheme 1). Results showed that EGFR monoclonal antibody-conjugated Au@Ag nanorod-DTNB nanoprobes (Au@Ag NR-nanotags) could be used for tumor boundary identification by targeting photoacoustic imaging and simultaneous enhanced SERS signal, and exhibit high photothermal conversion efficiency for photothermal therapy of gastric cancer. Prepared EGFR-Au@Ag NRs-DTNB nanoprobes have great potential in applications such as tumor boundary identification, targeted photoacoustic imaging and photothermal therapy of gastric cancer in near future.

\section{Experimental Section Materials}

Hydrogen tetrachloroaurate (III) trihydrate $\left(\mathrm{HAuCl}_{4} \cdot 3 \mathrm{H}_{2} \mathrm{O}\right)$, sodium borohydride $\left(\mathrm{NaBH}_{4}\right)$, silver nitrate $\left(\mathrm{AgNO}_{3}\right)$, ascorbic acid (AA), cetyltrimethylammonium bromide (CTAB), sodium hydroxide $(\mathrm{NaOH})$, bovine serum albumin (BSA), tween-20,5,5-dithiobis (2-nitrobenzoic acid) (DTNB), 1-ethyl-3-(3 dimethyl-aminopropyl) carbodiimide (EDC), glutaraldehyde were purchased from SigmaAldrich. Anti-EGFR antibody was purchased from Fisher. De-ionized water (Millipore Milli-Q grade) with a resistivity of $18.2 \mathrm{M} \Omega \cdot \mathrm{cm}$ was used in all the experiments.

\section{Raman spectra measurement}

Raman spectra were recorded at a small portable Raman spectrometer (BWS415, B\&W Tek Inc.) with an excitation wavelength of $785 \mathrm{~nm}$, a resolution of $5 \mathrm{~cm}^{-1}$, and a beam diameter of $10 \mu \mathrm{m}$. The integration time in each case was typically $20 \mathrm{~s}$.

\section{Synthesis and characterization of Au@Ag nanorods (Au@Ag NRs)}

Gold nanorods (Au NRs) were synthesized according to our previous report [14, 33]. Excess reagents were removed by centrifuging the asprepared Au NRs solution twice at $9100 \mathrm{rpm}$ for 10 min. The precipitate was re-dispersed in $1 \mathrm{~mL}$ of deionized water. Then, Au@AgNRs were synthesized using reported method with slight modification [37]. Typically, $1 \mathrm{~mL}$ of the purified Au NRs solution was added to $5 \mathrm{~mL}$ of $0.04 \mathrm{M}$ cetyltrimethyl ammonium bromide (CTAB) aqueous solution under vigorous stirring at room temperature. After that, $130 \mu \mathrm{L}$ of $0.1 \mathrm{M}$ ascorbic acid, $300 \mu \mathrm{L}$ of $10 \mathrm{mM}$ silver nitrate $\left(\mathrm{AgNO}_{3}\right)$ and $290 \mu \mathrm{L}$ of $0.1 \mathrm{M}$ sodium hydroxide $(\mathrm{NaOH})$ solution were added sequentially. The color of the solution changed gradually from brown to rose, indicating the formation of silver coating on $\mathrm{Au}$ NRs. Prepared Au@Ag nanorods were characterized by HR-TEM, TEM images were obtained by using a JEOL JEM-2010 model at an accelerating voltage of $120 \mathrm{kV}$. 
Synthesis and characterization of Au@Ag NRDTNB

The Au@AgNR-DTNB nanoprobes were prepared according to the following procedure. First, $1 \mathrm{~mL}$ of the as-prepared Au@AgNRs solution was centrifuged twice at $8000 \mathrm{rpm}$ for $10 \mathrm{~min}$ to remove the excess reagents. The pellet was dispersed in $1 \mathrm{~mL}$ of deionized water. Then, $10 \mu \mathrm{L}$ of $2 \mathrm{mM}$ DTNB ethanol solution was added and the resultant mixture was shaken at room temperature. After 10 min incubation, the mixture was added $100 \mu \mathrm{L}$ BSA $(0.5 \%$ in deionized water) mixed with $25 \%$ glutaraldehyde (60:1) and was shaken at room temperature for $4 \mathrm{~h}$ followed by centrifugation ( $8000 \mathrm{rpm}, 5 \mathrm{~min})$. In order to remove the excess aldehyde groups, pellets were re-suspended in $1 \mathrm{ml}$ of $10 \mathrm{mM}$ glycine/10 mM sodium citrate $(\mathrm{pH}=8)$ at the room temperature for 30 minutes, Finally, the BSA encapsulated Au@ AgNRs were washed twice by centrifugation (6000 rpm $10 \mathrm{~min}$ ) and re-suspended in $1 \mathrm{mM}$ sodium citrate, and stored at $4{ }^{\circ} \mathrm{C}$. For bioconjugation and long time storage, we resuspended the DTNB-encapsulated Au@ AgNRs in $10 \mathrm{mM}$ borate buffer.

SERS activity of Au NRs and Au@Ag NRs were compared by adding same amount of SERS reporter DTNB molecules to an equal volume of the nanorods solution of same concentration. $10 \mu \mathrm{L}$ of $0.01 \mathrm{M}$ DTNB was respectively mixed with $1 \mathrm{ml}$ of nanorods solution orAu@Ag NRs solution and stirred overnight at room temperature. After purification, the SERS spectra were recorded.

\section{Preparation and characterization of EGFR monoclonal antibody conjugated Au@AgNRs- DTNB}

Prepared Au@AgNR-DTNB were conjugated with anti-EGFR monoclonal antibodies. The carboxylic acid groups of BSA were activated with $100 \mu \mathrm{L} 50 \mathrm{mM}$ $N$-(3-(dimethylamino)-propyl)- $N$ ' ethylcarbodiimide (EDC). Excess EDC was removed after $30 \mathrm{~min}$ incubation by centrifugation. The activated particles were resuspended in $10 \mathrm{mM}$ borate buffer and reacted with mouse monoclonal anti-EGFR antibodies at room temperature for $2 \mathrm{~h}$ and then overnight at $4{ }^{\circ} \mathrm{C}$. The resulted antibody conjugates (EGFR-Au@Ag NR nanotags) were washed twice by centrifugation to remove free antibody, and re-suspended in $10 \mathrm{mM}$ borate, $1 \%$ BSA and $0.05 \%$ Tween-20 and stored at $4{ }^{\circ} \mathrm{C}$ until further use. The samples of AuNRs, Au@ AgNRs,Au@AgNRs@BSA,Au@AgNRs@BSA-@
Anti-EGFR were diluted with $1.0 \mathrm{~mL}$ deionized water to the appropriate concentration, mixed well, then, their hydrodynamic diameters and Zeta potentials were determined by dynamic light scattering (DLS) using a Malvern Zetasizer Nano ZS90. The DLS instrument was operated under the following conditions: temperature $25^{\circ} \mathrm{C}$, detector angle $173^{\circ}$, incident laser wavelength $633 \mathrm{~nm}$ and laser power $5 \mathrm{~mW}$.

\section{MTT assay for cytotoxicity of as-prepared nanoprobes}

The effects of EGFR-Au@AgNRs nanotags on MGC 803 cells proliferation was determined by the MTT (3-(4,5-dimethylthiazol-2-yl)-2,5diphenyltetrazolium bromide) assay. MGC 803 cells $\left(10^{6} \mathrm{ml}^{-1}\right)$ were seeded onto 96-well plates $(100 \mu \mathrm{l} /$ well) and incubated for $24 \mathrm{~h}$ at $37^{\circ} \mathrm{C}$ under a $5 \% \mathrm{CO}_{2}$ atmosphere. Then, $20 \mu \mathrm{L}$ different concentration of EGFR-Au@AgNR-nanotags were added respectively and incubated for $24 \mathrm{~h}$. After that, $50 \mu \mathrm{L}$ of MTT solution (MTT buffer to dilution buffer 1:4) was added into to each well and the plates were incubated for 4 $\mathrm{h}$. The reaction was terminated by adding $150 \mu \mathrm{L}$ of DMSO after removing the supernatant medium. When the purple formazan crystals were resolved by DMSO, the absorbances of the wells at $490 \mathrm{~nm}$ were measured with a microplate reader. MGC 803 cells withoutAu@ AgNRs@BSA were used as a control.

\section{Prepared nanoprobes for target specificity of gastric cancer}

EGFR positive gastric cancer cells (MGC803) and EGFR negative MDA MB-435 cells were grown in RPMI 1640 medium supplemented with 10\% (v/v) fetal bovine serum (FBS) at $37^{\circ} \mathrm{C}$ in a humidified atmosphere with $5 \% \mathrm{CO}_{2}$. MGC803 cells and MDA MB-435 cells were grown in 8-well plates, incubated with EGFR-Au@Ag NR nanotags (125 pM) for 1 h at $37^{\circ} \mathrm{C}$, washed with PBS for 3 times, then SERS measurements were done.

\section{Prepared nanoprobes for enhanced SERS detection of tumor boundary of gastric cancer in vivo}

All animal experiments were approved by the Institutional Animal Care and Use Committee of Shanghai Jiao Tong University (SCXK-2012-0002). $100 \mu \mathrm{L}$ of bothAu@AgNRs-DTNB and AuNRsDTNB were respectively injected to a gastric cancerbearing nude mice model, and then SERS spectra were measured at the sites of injection under $808 \mathrm{~nm}$ NIR 
laser irradiation for $50 \mathrm{~s}$.

\section{Prepared nanoprobes for targeted photo- acoustic imaging}

EGFR-Au@Ag NR nanotags for optoacoustic imaging of local tumor tissues in gastric cancer-bearing nude mice model were done by using multispectral optoacoustic tomography:

(a) Spectrometer measurement: An absorption spectrum from each solution used was recorded on an OceanOptics CUV-UV + USB2000 spectrometer and processed with SpectraSuite (OceanOptics, Ostfi ldern, Germany) using a $1 \mathrm{~mL}$ sample cuvette, using either PBS pure as a buffer or PBS containing $1 \% \mathrm{w} / \mathrm{w}$ bovine serum albumin, as mentioned. Around $18.6 \mu \mathrm{g} \mathrm{mL}^{-1}$ AuNPrs was used to obtain 0.5 OD. As a reference, Ntracker gold nanorods absorbing at $808 \mathrm{~nm}$ (Nanopartz, Loveland, CO, USA) were used at $18 \mu \mathrm{g} \mathrm{mL}^{-1}$ (0.5 OD).

(b) Phantom experiments: Cylindrical phantoms with a diameter of $2 \mathrm{~cm}$ were prepared using a gel made from distilled water. The gel contained Agar (Sigma-Aldrich, St. Louis, MO, USA) for jellification (1.3\% w/w) and an intralipid 20\% emulsion (SigmaAldrich, St. Louis, MO, USA) for light diffusion (6\% $\mathrm{v} / \mathrm{v})$, resulting a reduced scattering coeffi cient $(\mu \mathrm{S})$ of $c a .10 \mathrm{~cm}^{-1}$. A cylindrical inclusion containing a sample of ca. $3 \mathrm{~mm}$ in diameter was placed in the middle of the phantom, along with a tube containing classical black ink with an optical density of 0.2 for intensity measurement references.

Imaging of the phantoms was done in the previously described MSOT system using transversal plane imaging at a single position, located approximately in the middle of the phantom. Data acquisition was performed using 20 averages and at each wavelength between $680 \mathrm{~nm}$ and $900 \mathrm{~nm}$ in steps of $5 \mathrm{~nm}$.

(c) In vivo experiments: Procedures involving animals and their care were conducted in compliance with institutional guidelines, and with the approval of Shanghai jiaotong university (SCXK-2012-0002). Female $\mathrm{CD}^{-1}$ nu/nu mice were subcutaneously inoculated with approximately 1.5 million MGC 803 gastric cancer cells in $15 \mu \mathrm{L}$ BD Matrigel (BD Biosciences, Bedford, USA), at a position located between the kidneys and the base of the tail. For the imaging experiments, $100 \mu \mathrm{L}$ of the prepared EGFR-Au@Ag NRs (0.744 mg mL ${ }^{-1}$ ) was injected intravenously in the tail vein after anesthesia using 2\% isoflurane in oxygen. Anesthesia was maintained at
$1.8 \%$ isoflurane in oxygen throughout the acquisitions. Imaging was performed in the previously described MSOT system using transversal slices with a 1 $\mathrm{mm}$ step at the tumor, liver and kidney region, at a wavelength of $680,710,740,770,800,830,860 \mathrm{~nm}$ for each position, using 50 averages per wavelength in order to minimize the influence of animal movement in the images. Animal were sacrificed by cervical dislocation and stored at $-80{ }^{\circ} \mathrm{C}$ for further analysis. Heart, liver, spleen, lung, kidney, intestinal tissues and brain were removed and then fixed in $4 \%$ neutral buffered formalin for at least 1 day. The samples were then dehydrated in an ethanol series, processed into paraffin, and sectioned. Then, H\&E (hematoxylin and eosin) staining was carried out in accordance with the standard protocol to monitor the morphological features of each organ.

\section{Biodistribution of prepared nanoprobes and local photothermal therapy}

Animal experiments were performed according to Guidelines for Animal Care and Use Committee, Shanghai Jiao Tong University. All the mice used in this study were complied with current ethical considerations: Approval (SYXK-2007-0025) of the Institutional Animal Care and Use Committee of Shanghai JiaoTong University (Shanghai, China). MGC803 cells $\left(5 \times 10^{6}\right)$ were injected subcutaneously into the right rear flank area of 50 female nude mice of age 6 to 8 weeks. When tumors grew to $5 \mathrm{~mm}$ in diameter, $200 \mu \mathrm{g}$ of pepared nanoprobes was injected into mice via tail vein. For the blocking experiment, ten mice were injected with the mixture of $0.5 \mathrm{mg}$ of PBS. For targeting experiment, $200 \mu \mathrm{g}$ of pure Au@ Ag NRs was injected into ten nude mice via tail vein. Mice were respectively sacrificed at $3 \mathrm{~h}, 6 \mathrm{~h}, 9 \mathrm{~h}$, and $12 \mathrm{~h}$. Blood and organs were collected and kept in liquid nitrogen. Blood and tissue samples were lysed in aqua regia. The precipitates were dissolved in 0.5 $\mathrm{M} \mathrm{HCl}$, the amount of Au@Ag NRs was measured by inductively coupled plasma mass spectrometry (Thermo, U.K.).

The nude mice model with subcutaneous gastric cancer were prepared as mentioned above. For photothermal experiments, the $808 \mathrm{~nm}$ laser trigger was setup as $1 \mathrm{w} / \mathrm{cm}^{2}$ for $40 \mathrm{~s}$; the nude mice models were irradiated for one time per day, and then raised for 5 weeks. The tumor volumes were calculated over 5 weeks; the tumor growth weekly was monitored with a caliper and the tumor volume was calculated according 
to the formula: $\mathrm{V}=4 / 3 \times \pi \times(\mathrm{a} / 2) 2 \times(\mathrm{b} / 2)$, where $\mathrm{a}$ is the length and $b$ is the width diameter of the tumor, the tumor growth curve was drawn.

\section{Statistical analysis}

All experiments were performed in triplicate unless otherwise indicated. Results were expressed as mean values \pm standard deviation. Statistical differences were calculated by a two-tailed, unpaired Student's t-test at a significance level of $p<0.05$.

\section{Results and Discussion Preparation and characterization of Au@Ag nanorods}

Plasmonic Au@Ag nanorods were prepared by using our previous modified method [14, 40]. The synthesis procedure is shown in Fig. S1. As shown in Fig. 1, prepared Au@Ag nanorods were 45 nm in length and $15 \mathrm{~nm}$ in width; the silver thin layer was 2 $\mathrm{nm}$ in thick; the UV-VIS absorption peaks respectively
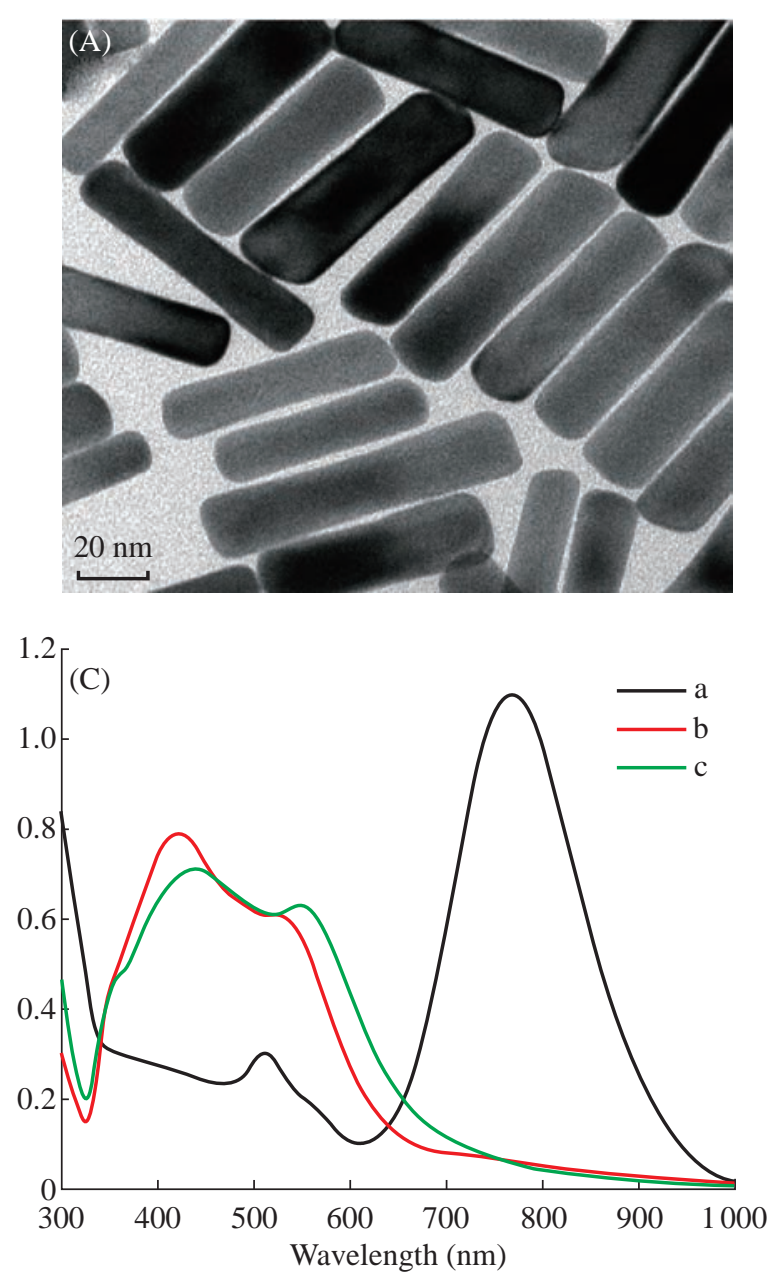

located in $520 \mathrm{~nm}$ and $756 \mathrm{~nm}$; XRD analysis showed that preparedAu@Ag NRs mainly are composed of Au and silver element. The high resolution TEM pictures of Au nanorods,Au@Ag nanorods, Au@Ag NRDTNB, EGFR-Au@Ag NR-DTNB were shown in Fig. S2.

\section{Preparation and characterization of Au@ AgNR-DTNB}

PreparedAu@Ag nanorods were mixed with raman reporter DTNB, then the SERS signal was detected. As shown in Fig. 2, the SERS spectra of DTNB are distinguished by the signature peaks at $845,1063,1150$, 1332 and $1556 \mathrm{~cm}^{-1}$. The SERS intensity of the Au@ AgNR-DTNB mixture is higher than that of the Au NR-DTNB mixture, showing excellent $1 \mathrm{~L}$ fold enhancement of SERS signal after gold nanorods were modified with silver coating, which can be attributed to the electronic ligand effect in core-shell bimetallic nanoparticles [41]. Raman spectra of $100 \mu \mathrm{L}$ Au@Ag NRs mixed with $1 \mu \mathrm{L}$ different concentration of DTNB
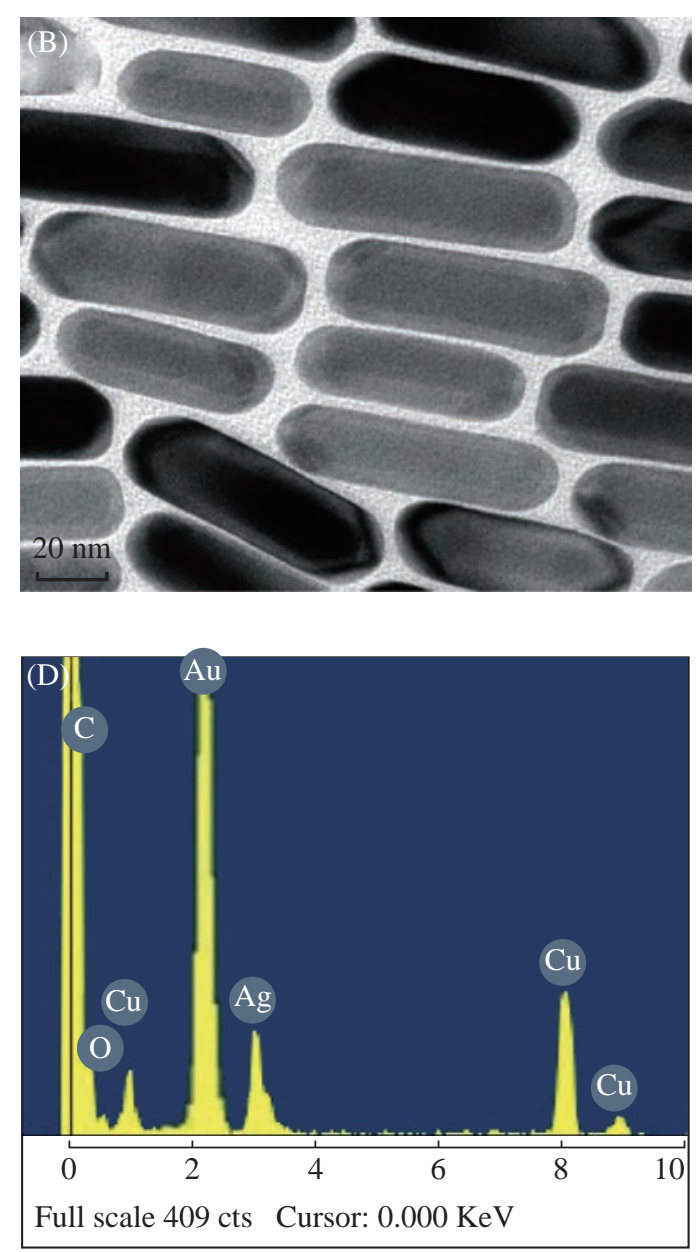

Fig. 1 Characterization of Au@Ag NRs. TEM images of (A) Au NRs, (B) Au@Ag NRs. (C) UV absorption spectra of (a) Au NRs, (b) Au@AgNRs, and (c)Au@Ag@BSA. (D) XRD spectra of Au@Ag NRs. 

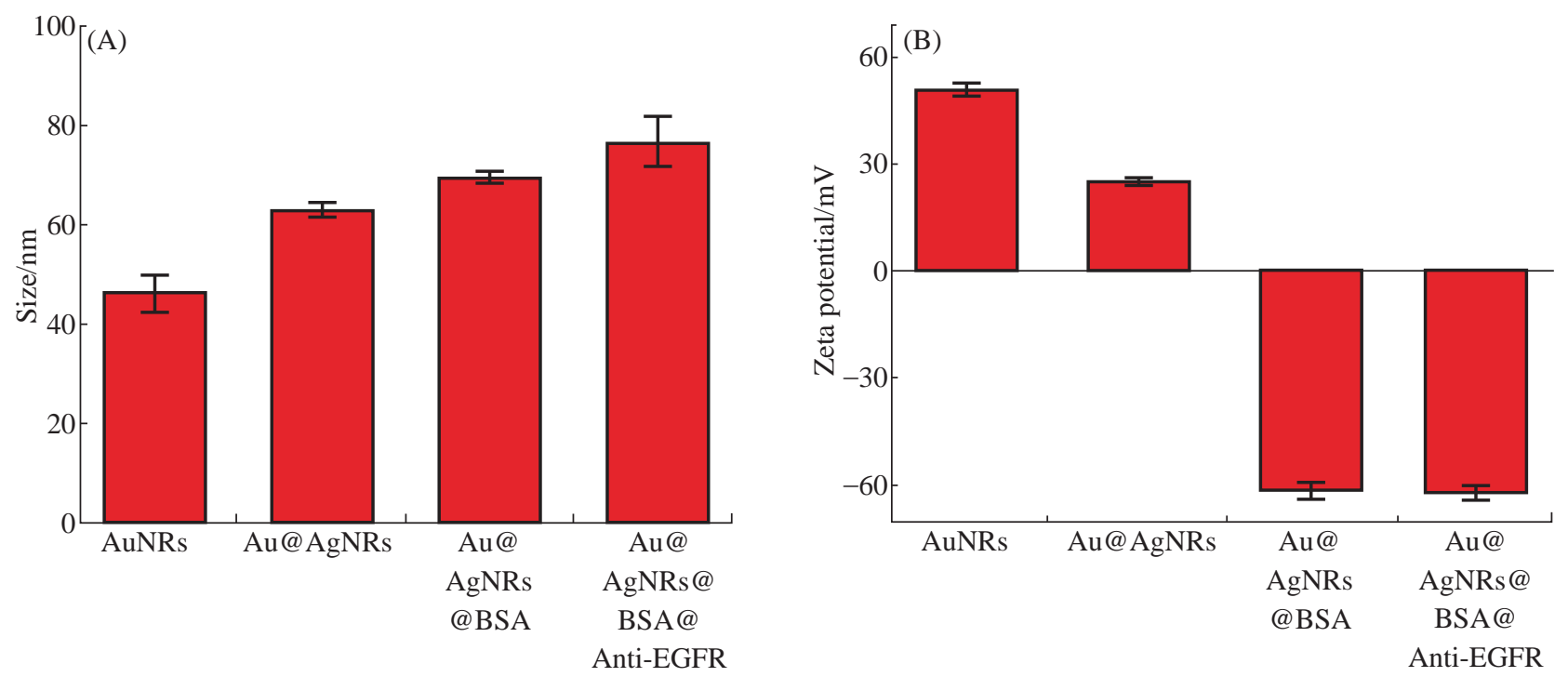

Fig. 2 (A) The size and (B) potential of AuNRs, Au@AgNRs, Au@AgNRs@BSA, EGFR-Au@AgNR-nanotags.

(10 $\mu \mathrm{M}, 100 \mu \mathrm{M}, 1 \mathrm{mM}, 2 \mathrm{mM}, 10 \mathrm{mM})$ was shown in Fig. S3.

We also repeated the experiments for 10 times, the results were shown in Fig. S4, demonstrating Au@Ag nanorods-based SERS own good repeatability. This result shows that Au@Ag NRs can be used for SERS based biomarker ultrasensitive detection.

\section{Preparation and characterization of EGFR- Au@Ag NR-nanotags}

Epidermal growth factor receptors play an important role in cell proliferation and growth and exhibit upregulation expression in gastric cancer $[42,43]$. To prepare theAu@Ag NR-DTNB nanoprobes that could selectively target gastric cancer cells with over-expressed EGFR, we used BSA modified Au@ Ag NR-DTNB, and then conjugated with antiEGFR monoclonal antibody (as shown in Supporting data Fig. S1). The resultant EGFR-Au@Ag NRsDTNB nanoprobes(EGFR-Au@Ag NR nanotags) are characterized. As shown in Fig. 3, prepared EGFRAu@Ag NR nanotags were 80 nm in diameter by dynamic light scattering, zeta potential of EGFR-Au@ Ag NR-DTNB nanoprobes were $-60 \mathrm{~V}$.

\section{Cytotoxicity and targeting ability of EGFR- Au@Ag NR nanotags}

As shown in Fig. 4, prepared EGFR-Au@AgNR nanotags did not exhibit the obvious cytotoxicity to MGC 803 cells within the scope of 2 nM [44-46]. EGFR positive gastric cancer MGC803 cells and EGFR negative MDA MB-435 cells were co-incubated

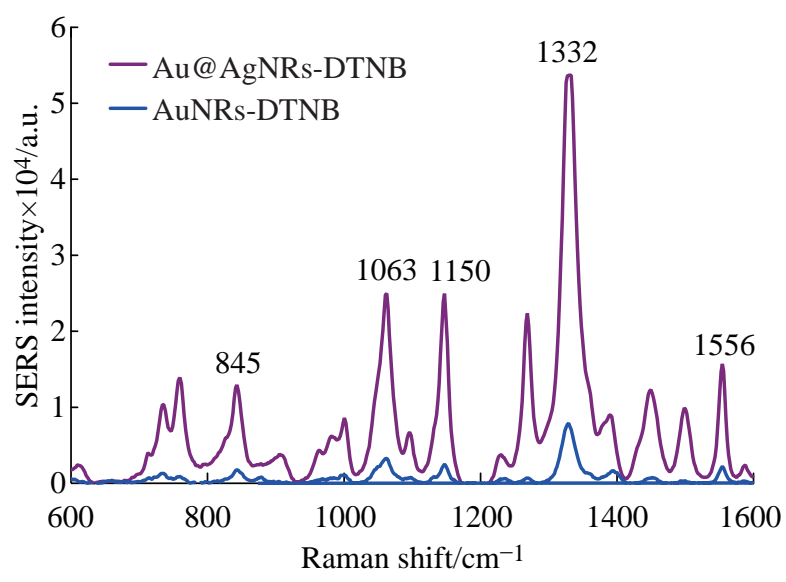

Fig. 3 SERS spectra of DTNB labeled Au NRs and Au@ AgNRs.

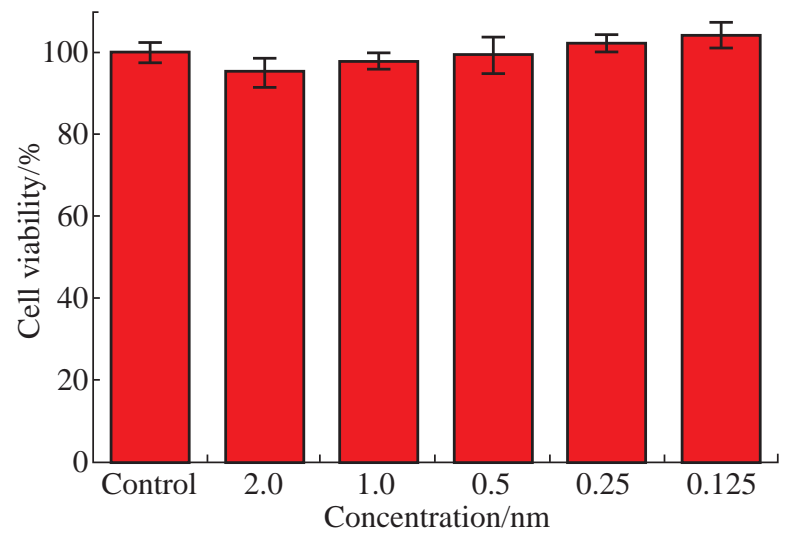

Fig. 4 Viability of MGC 803 cells exposed to 2.0, 1.0, 0.5, 0.25, 0.125 nM of EGFR-Au@AgNRs-DTNB for 24 h, and MGC 803 cells without nanoprobes were used as a control.

with 250 pM prepared EGFR-Au@AgNRs-DTNB nanoprobes for $1 \mathrm{~h}$ at $37^{\circ} \mathrm{C}$; then cells were washed 
with PBS for 3 times, and then SERS measurements were done as shown in Fig. S4. As shown in Fig. 5, the 10 fold enhancement of SERS signal were observed in MGC803 cells incubated with prepared EGFR-Au@ AgNRs-DTNB, at the same time, very weak signals could be observed in MDA MB-435 cells incubated with prepared EGFR-Au@AgNR nanotags, which fully demonstrate that prepared EGFR-Au@AgNR nanotags can specifically target and combine with gastric cancer MGC 803 cells. As shown in Fig. 5(C), prepared EGFR-Au@AgNRs-DTNB nanoprbes could enter into cytoplasm of gastric cancer.

\section{EGFR-Au@AgNR nanotags for SERS-based tumor boundary identification}

To investigate the feasibility of use of EGFR-Au@ AgNR nanotags as tumor boundary identification, 100 $\mu \mathrm{L}$ of bothAu@AgNRs-DTNB and AuNRs-DTNB respectively were injected to subcutaneous gastric cancer-bearing nude mice model for $1 \mathrm{~h}$. The SERS spectra at the sites of injection was measured under a $808 \mathrm{~nm}$ NIR laser irradiation. As shown in Fig. 6, the SERS signal from the tumor site injected with Au@ AgNRs-DTNB were markedly higher than the signal from the tumor site injected with AuNRs-DTNB, which fully demonstrate that prepared Au@AgNRsDTNB can be used for identification tumor boundary. We also repeated the whole experiment, and found the SERS signal can be obtained with high repeatability ( supporting data Fig. S5).

Then, $250 \mu$ g prepared EGFR-Au@AgNR nanotags were injected into gastric cancer-bearing nude mice via tail vein for 4h, as shown in Fig. 7. The SERS imaging can be obtained clearly from gastric cancer site; the result highly suggests that prepared EGFR-Au@AgNR nanotags can target in vivo gastric cancer tissues, accumulate in tumor sites, and can be used to identify in vivo gastric cancer boundary.

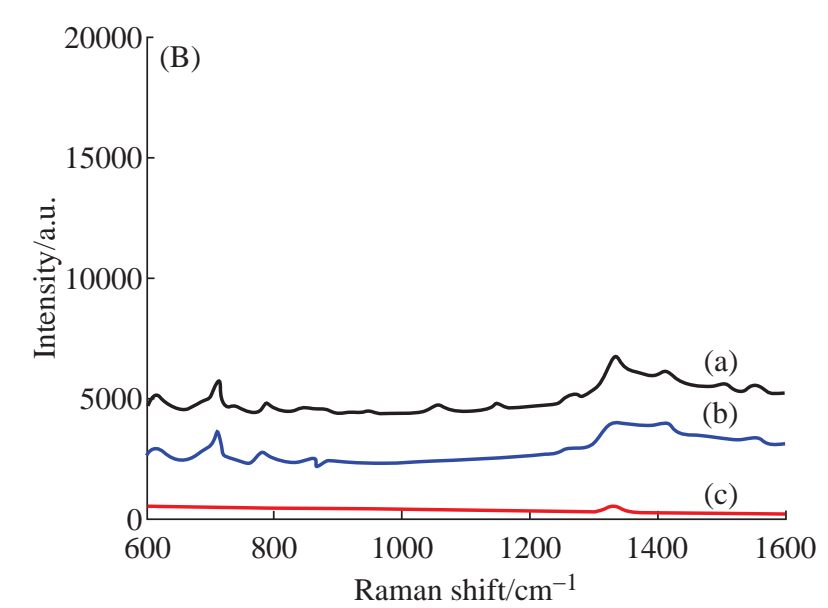

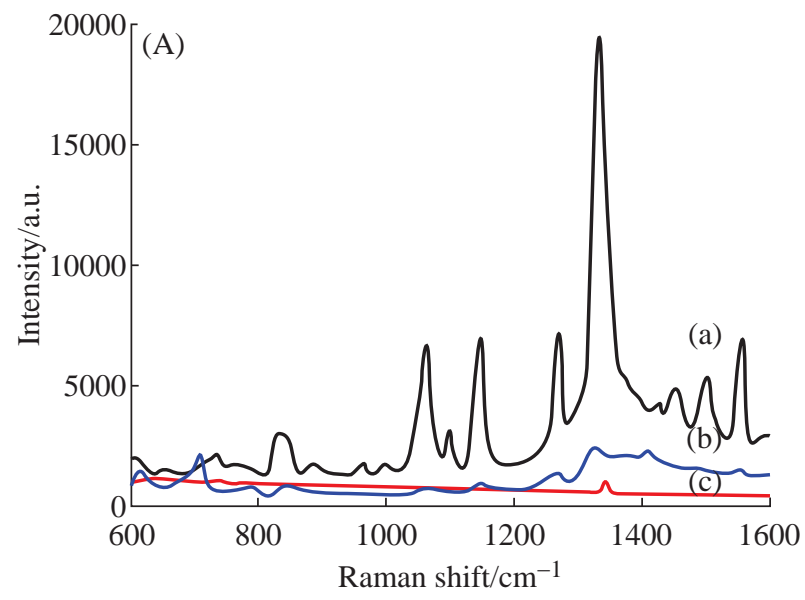

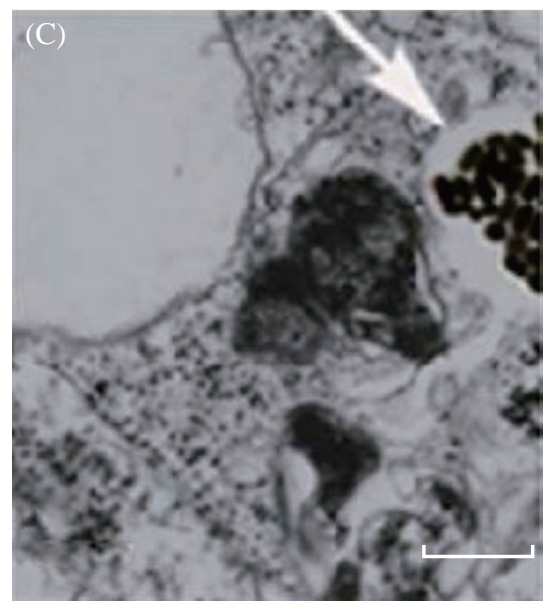

Fig. 5 SERS spectra showing EGFR targeted detection of tumor cells. (A) SERS signals from the MGC 803 cells treated with (a) anti-EGFR conjugated Au@AgNR@BSA-DTNB, (b) Au@AgNR@BSA-DTNB, and (c) SERS signal of just MGC803 cells. (B) SERS signals from the MDA 435 cells (a) treated with Anti-EGFR conjugated Au@AgNR@BSA-DTNB, (b) Au@AgNR@BSADTNB, and (c) SERS signal of just MDA 435 cells. (C) High resolution TEM picture of Au@Ag NR nanotags in cytoplasm of MGC 803 cell (scale bar: $100 \mathrm{~nm}$ ). 


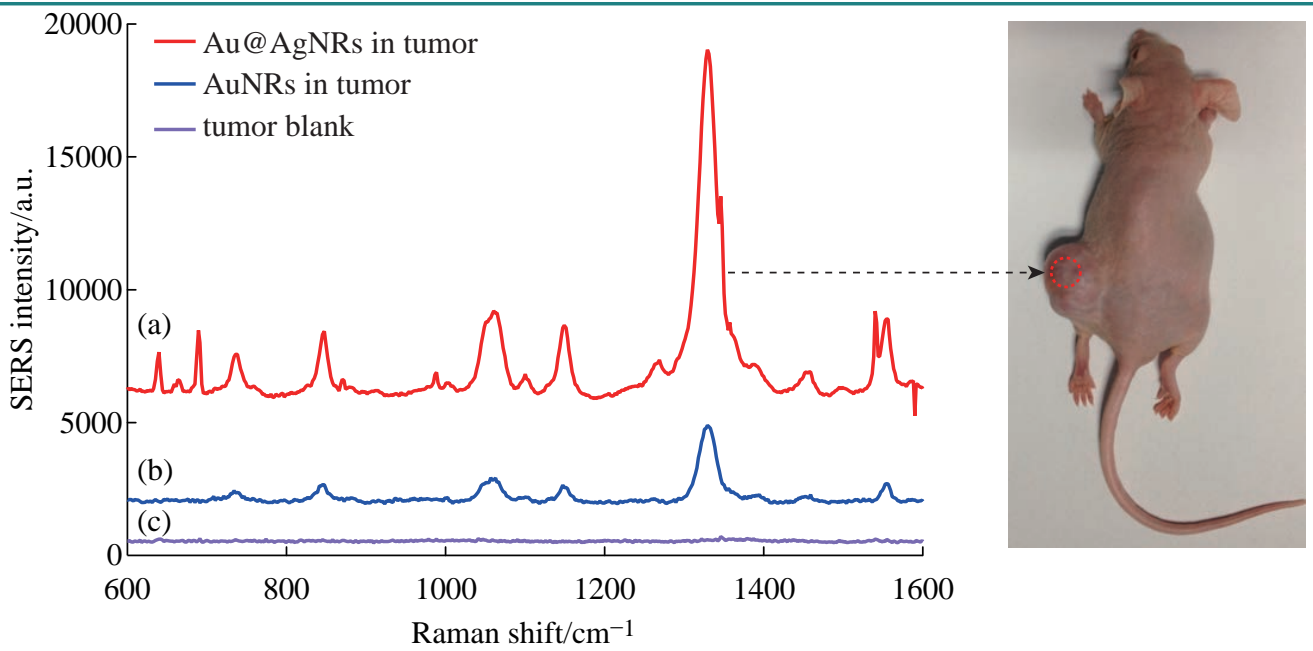

Fig. 6 In vivo SERS spectra obtained from BSA coated (a) Au@AgNRs-DTNB and (b) AuNRs-DTNB. Nanoparticles were injected into stomach tumor. The injection sites and laser beam positions are indicated by circles on the animal. The nude mouse received 100 ul of the SERS nanoparticles tags $(50 \mathrm{pM})$ by subcutaneous (1-2 mm under the skin). The subcutaneous spectrum was obtained in $10 \mathrm{~s}$, and the control spectrum (obtained in an area away from the injection site) also in 10 s. The spectral intensities are adjusted for comparison by a factor as indicated. These features are distinct from (c) the animal tumor Raman signals. Excitation wavelength, 785 nm; laser power, $20 \mathrm{~mW}$.
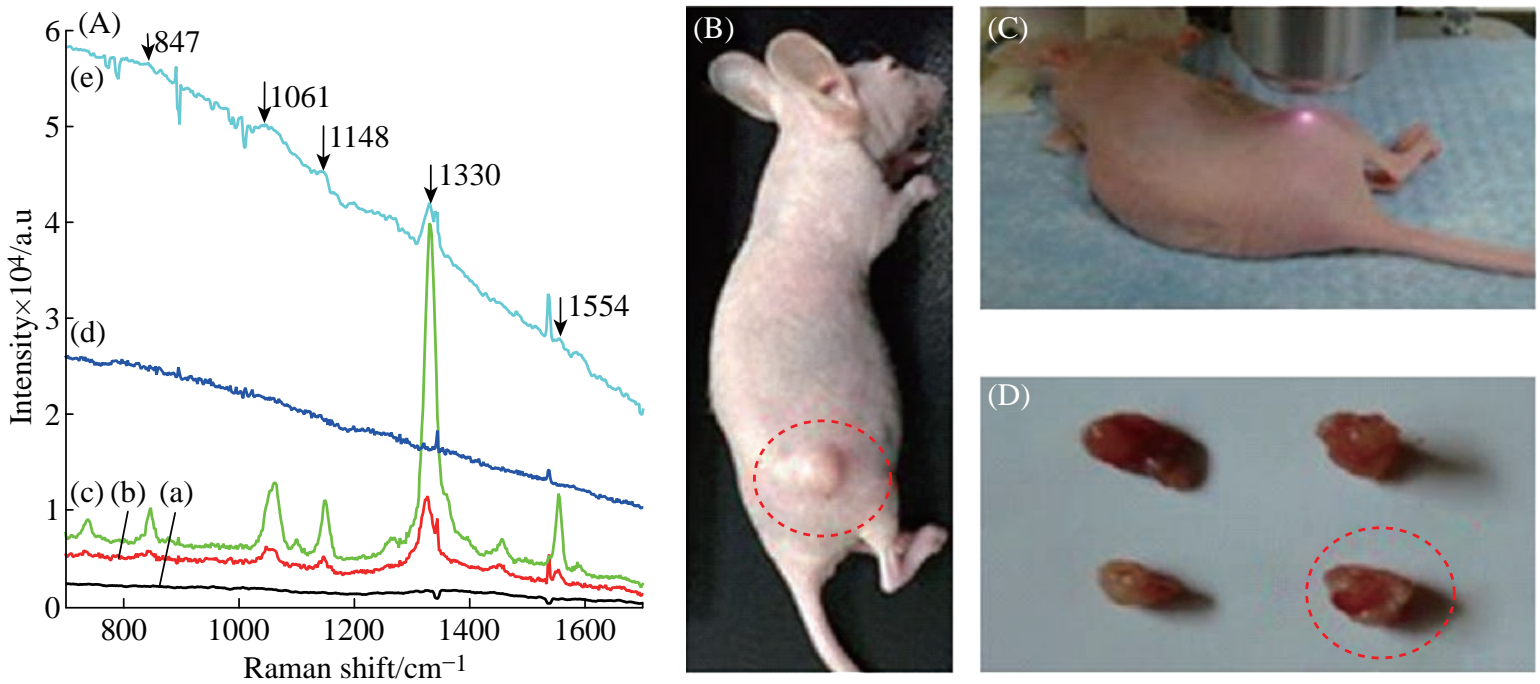

Fig. 7 (A) SERS spectra of AuNRs, Au@Ag NRs, EGFr-Au@Ag NRs mix with DTNB under 1 W/cm² Laser irradiation: (a) animal control; (b) AuNPs; (c) Au@AgNPs; (d) gastric control; and (e) EGFR-Au@Ag NRs in gastric cancer tissues collected time 30 s. (B) MGC803 gastric cancer-bearing nude mouse model. (C) Tumor site under $1 \mathrm{~W} / \mathrm{cm}^{2}$ laser irradiation. (D) Collected tumor tissues for nude mice models.

\section{EGFR-Au@AgNR nanotags for targeted photoacoustic imaging}

In order to visualize EGFR-Au@AgNR nanotags in vivo, gastric cancer-bearing nude mice model were prepared. We also compared the light absorbance of Au NRs, Au@Ag NRs, Hb and $\mathrm{HbO}_{2}$ by spectrophotometer, as shown in Fig. 8, Au@Ag NRs owns strongest absorption peak, locates in $756 \mathrm{~nm}$. Finally, we selected $808 \mathrm{~nm}$ laser as the light source. The prepared EGFR-Au@AgNRs-DTNB nanoprobes were injected into gastric cancer-bearing nude mice via tail vein; the photoacoustic signal were obtained by MSOT apparatus. As shown in Fig. 9. At 4 h postinjection, tumor boundary became clearer; at $8 \mathrm{~h}$ post-injection, the tumor boundary became clearest, which fully demonstrate that prepared nanoprobes can identify tumor boundary by photoacoustic imaging, which should be the first report.

\section{EGFR-Au@AgNRs-DTNB nanoprobes for targeted photothermal therapy}

As shown in Fig. 10(A), prepared Au@Ag NRs can produce high temperature as laser irradiation 


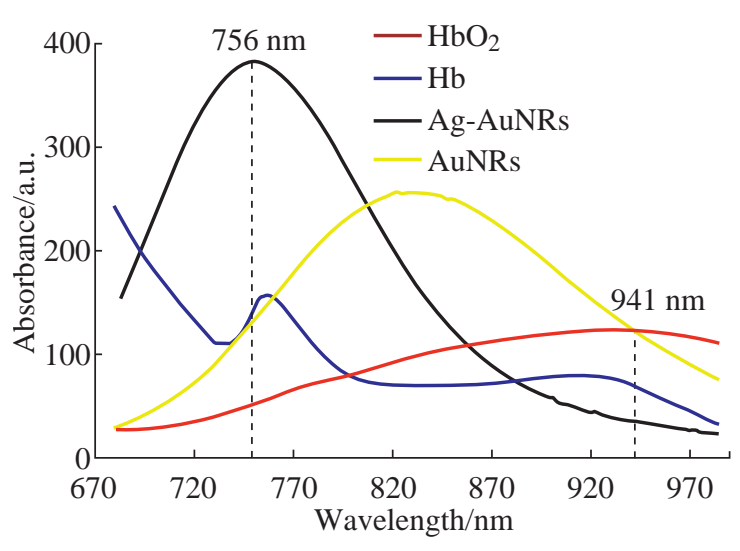

Fig. 8 Comparison of the light absorbance of Au NRs, Au@Ag $\mathrm{Nrs}, \mathrm{Hb}, \mathrm{HbO}_{2}$ by spectrophotometer.

time increases. Under $1 \mathrm{w} / \mathrm{cm}^{2}$ power laser irradiation for $30 \mathrm{~s}$, tumor local temperature reached to $40^{\circ} \mathrm{C}$; at $3 \mathrm{~min}$ after irradiation, local temperature reached almost $100^{\circ} \mathrm{C}$. Compared with only Au nanorods, their photothermal conversion efficiency existed statistical difference $(P<0.01)$, which fully demonstrate that silver film layer can enhance markedly photothermal conversion efficiency of Au NRs. Regarding the suitable temperature of photothermal therapy, there is some controversies. High temperature such as more than $70^{\circ} \mathrm{C}$ is used for local tumor treatment, which owns obvious advantages as follows: (a) high heat energy within 1 min quickly destroys and kills tumor cells, quickly forms coagulum, and decreases the chance of tumor cell metastasis; (b) high heat energy does not damage neighbour organs or tissues because of too short time; (c) tumor local coagulum can be degraded and slowly absorbed; and (d) finally local tumor tissues disappear [47-50]. Clinical therapeutic experiences also confirm this point. Conversely, lower temperature such as less than $45^{\circ} \mathrm{C}$, in the course of destroying tumor cells, improve blood circulation in tumor site, and speed up tumor cell micrometastasis. Finally, we selected $1 \mathrm{w} / \mathrm{cm}^{2}$ power $808 \mathrm{~nm}$ laser irradiation for $70 \mathrm{~s}$ as the local photothermal treatment condition for in vivo gastric cancer.

As shown in Fig. 11, EGFR-Au@AgNRs-DTNB nanoprobes remarkably inhibit growth of gastric cancer by photothermal therapy, compared with other groups, there existed statistical difference $(P<0.01)$, which fully demonstrate that prepared nanoprobes can be used for targeted photothermal therapy of in vivo gastric cancer.
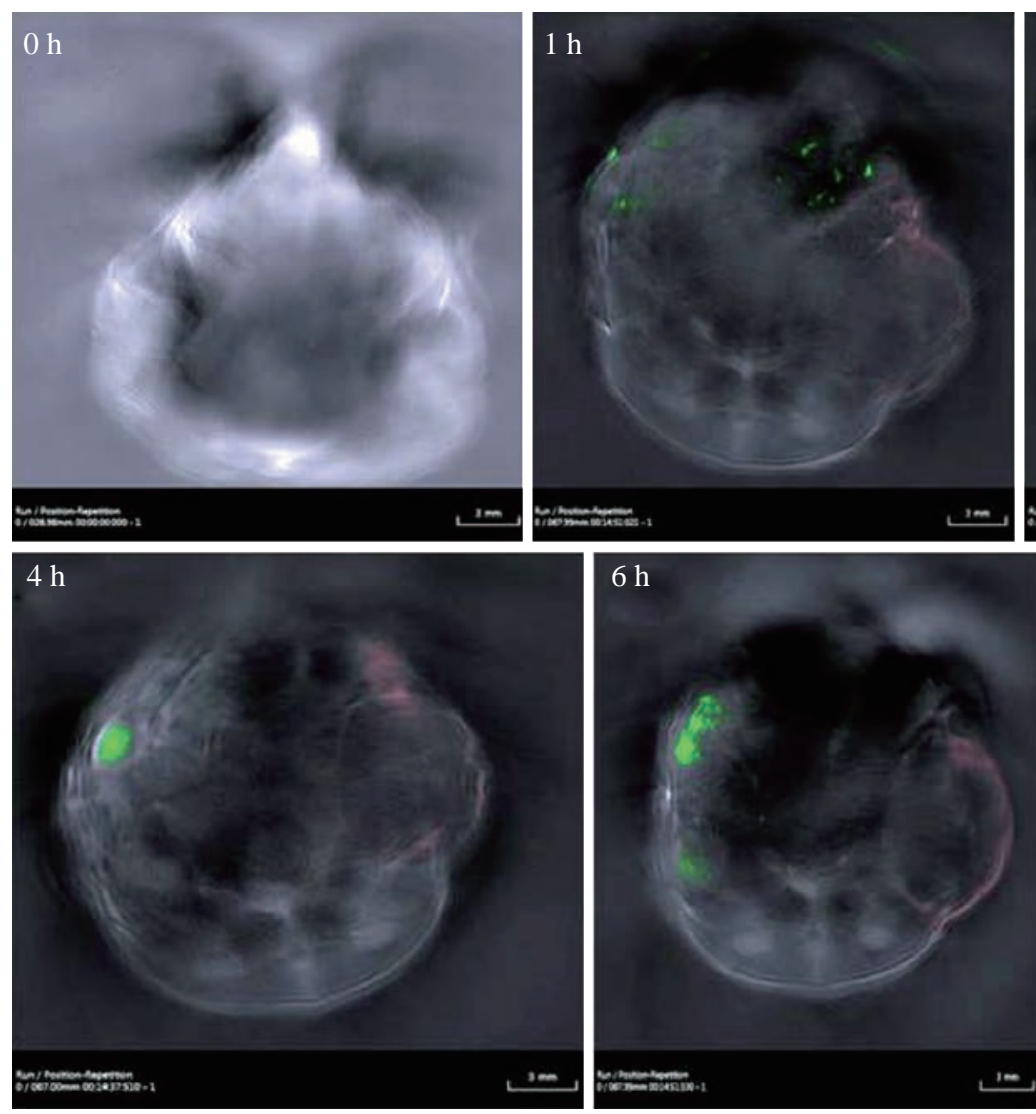

Fig. 9 Photoacoustic imaging of gastric cancer site at 0 h, 1 h, 2 h, 4 h, 6 h, 8 h post-injection by OMST. 

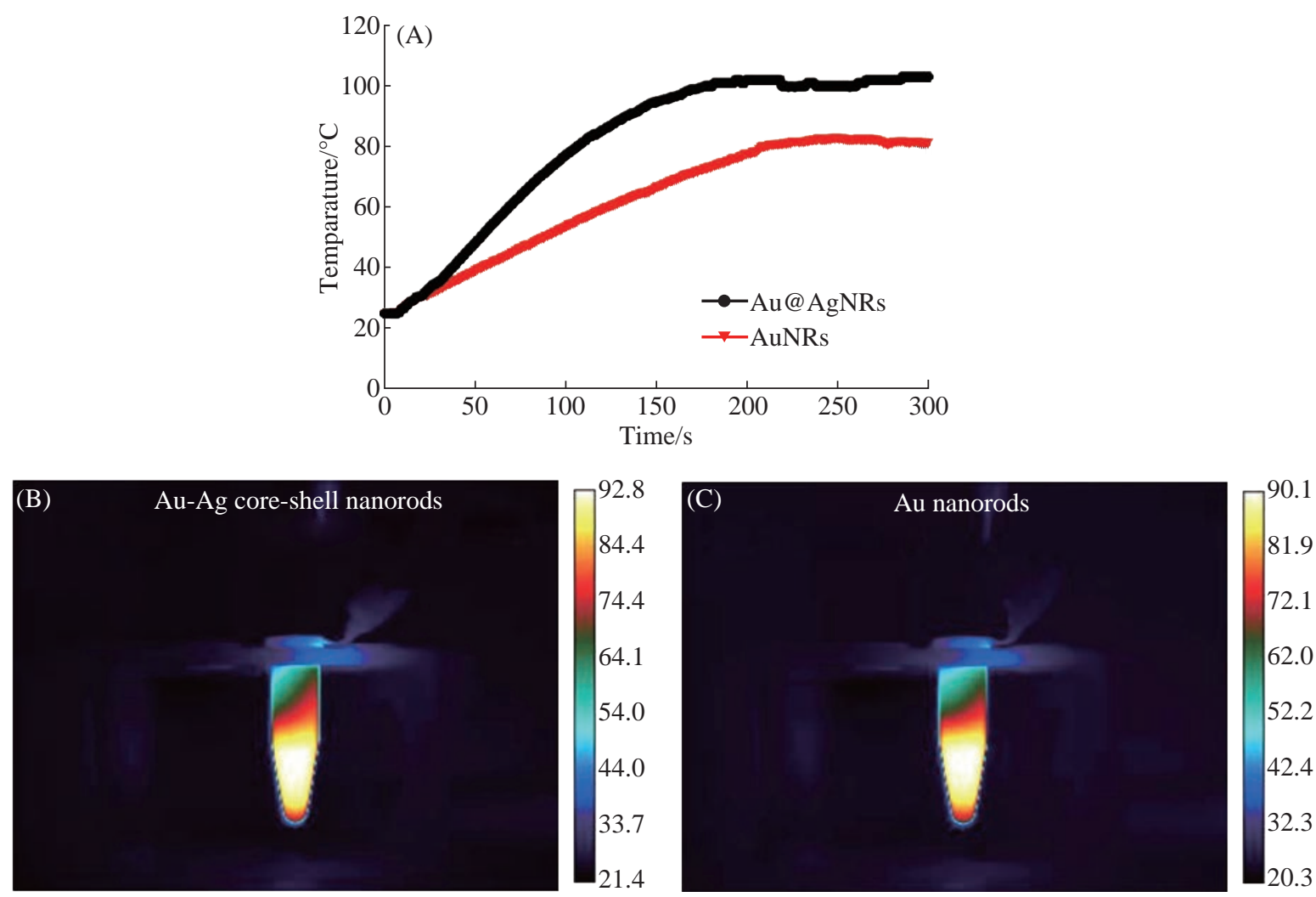

Fig. 10 Time-temperature curve of prepared Au@Ag nanorods and Au nanorods under $1 \mathrm{w} / \mathrm{cm}^{2} 808$ nm laser irradiation. (A) Timetemperature curve of prepared Au@Ag nanorods, temperature is $100{ }^{\circ} \mathrm{C}$ under $25 \mathrm{~W} 808 \mathrm{~nm}$ laser irradiation for $180 \mathrm{~s}$. (B) Real time temperature of prepared Au@Ag nanorods under 25 W laser irradiation for 150 s. (C) real time temperature of prepared Au nanorods under $25 \mathrm{~W}$ laser irradiation for $150 \mathrm{~s}$.

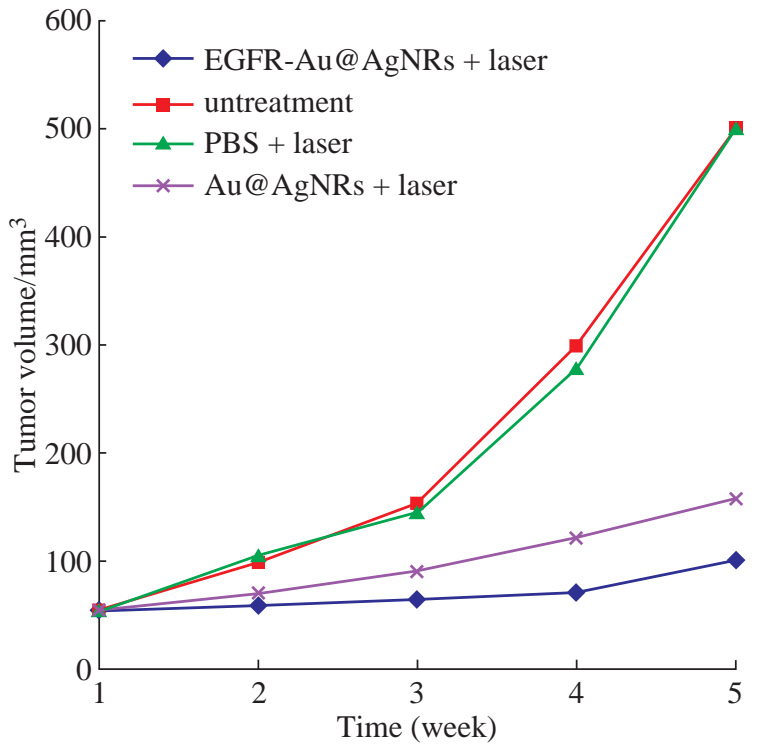

Fig. 11 Tumor volume-time growth curve.

\section{Distribution of EGFR-Au@AgNRs nanotags and pathological analysis}

As shown in Fig. 12, at 2 h post-injection, 10\% or so prepared nanoprobes accumulated in tumor sites; at $4 \mathrm{~h}$ post-injection, $18 \%$ or so prepared nanoprobes located in tumor sites; at $6 \mathrm{~h}$ post-injection, 20\% prepared nanoprobes located in tumor sites; at $8 \mathrm{~h}$ post-injection, $22 \%$ or so prepared nanoprbes located in tumor sites. Almost 15\% prepared nanoprobes located in liver and spleen tissues, at $6 \mathrm{~h}$ post-injection, especially interested phenomena, prepared nanoprobes also located in intestinal tissues. The amount in intestinal tissues reached to $20 \%$, more than the amounts in liver and spleen tissues. We also observed that the amount of prepared nanoprobes in brain tissues can not be detected, which means that prepared nanoprbes can not get through blood-brain barrier, and enter into brain tissues.

We also examined important organs by HE staining method, as shown in Fig. 13, we did not find that prepared nanoprobes could cause lesion of important organs such as heart, brain, lung, liver, spleen, kidney, which highly suggest that prepared nanoprobes own good biocompatibility.

\section{Conclusions}

In summary, we have developed a novel theranostic 


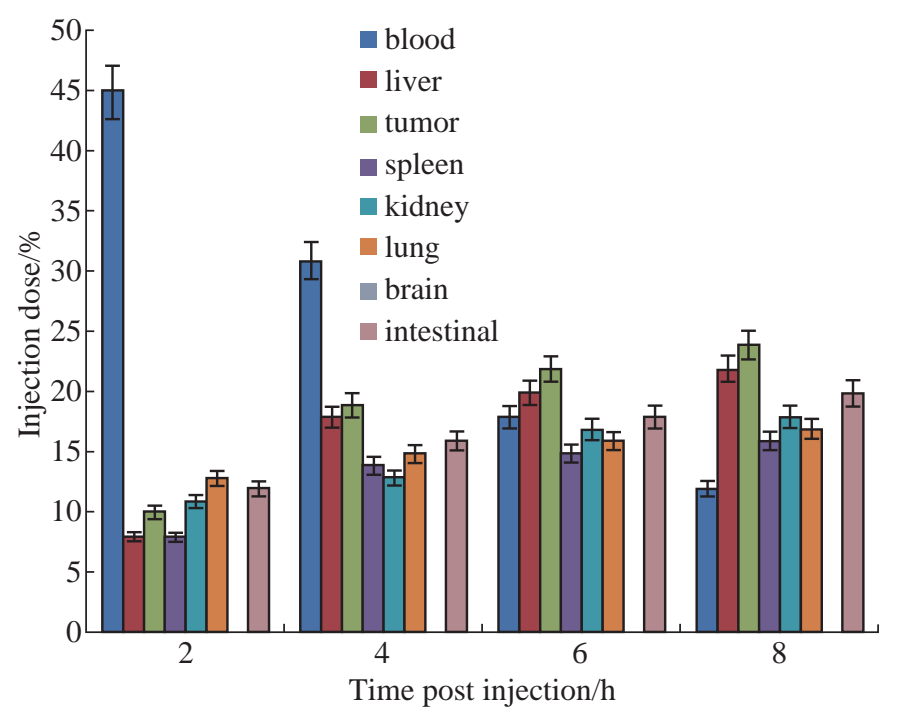

Fig. 12 Biodistribution of EGFR-Au@ Ag NR-nanotags at 2 h, 4 h, 6 h, 8 h post-injection.
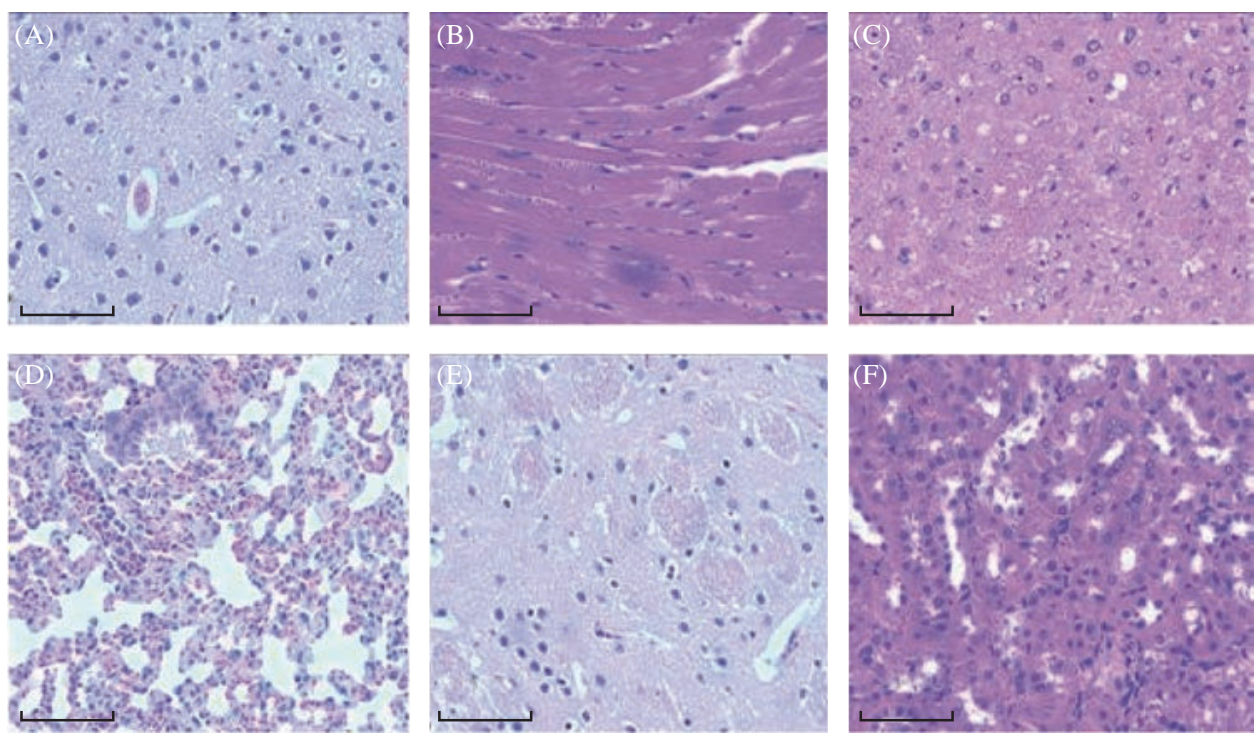

Fig. 13 Histological imagines $(20 \times)$ of different tissues using hematoxylin and eosin staining: (A) brian; (B) heart; (C) liver; (D) lung; (E) kidney; (F) spleen (bar: $10 \mu \mathrm{m}$ ).

platform based on EGFR antibody-conjugated Au@ Ag NRs-DTNB nanoprobes for targeted photoacoustic imaging ,tumor boundary identification, and local photothermal therapy of in vivo gastric cancer. More importantly, when we selected DTNB as Raman molecules, EGFR antibody-conjugated Au@Ag NRDTNB markedly enhance SERS imaging. Using SERS imaging, we could identify clearly the tumor boundary. We also observed that prepared nanoprobes can enhance photoacoustic imaging, and can also display tumor boundary clearly. Using photoacoustic imaging to identify tumor boundary should be the first report, which can be used to guide operation therapy, and own clinical translational prospect.

We also observed that silver thin layer markedly enhanced Au nanorods photothermal conversion efficiency. Under $1 \mathrm{w} / \mathrm{cm}^{2} 808 \mathrm{~nm}$ laser irradiation for 3 min, prepared Au@Ag NRs can produce local temperature up to $100{ }^{\circ} \mathrm{C}$. Up to date, it should be the highest temperature produced by Au nanorods-based nanocomposites. In order to avoid burn damage of peripheral tissues around tumor site caused by high temperature, we selected $70 \mathrm{~s}$ as the laser irradiation time. We achieved good therapeutic efficacy, the growth of tumor were markedly inhibited by local photothermal therapy.

We also investigated the distribution of in vivo prepared nanoprobes. At $6 \mathrm{~h}$ post-injection, nanoprobes mainly accumulated in the tumor sites; remaining nanoprobes also distributed in liver, spleen, kidney, 
intestinal tissues, and did not produce serious damages to important organs. Therefore, prepared nanoprobes own good biocompatibility, and display clinical translational prospect. How to realize all prepared nanoprobes accumulate in tumor sites has become our further study aim. High performance EGFR antibodyconjugatedAu@Ag NRs bimetallic nanoprobes own great potential in applications such as targeted photoacoustic imaging, tumor boundary identification, and photothermal therapy of in vivo gastric cancer in near future.

\section{Acknowledgements}

This work was supported in part by the Intramural Research Program (IRP), National Institute of Biomedical Imaging and Bioengineering (NIBIB), National Institutes of Health (NIH), China National Key Basic Research Program (973 Project) (Nos. 2010CB933902 and 2015CB931802), National Natural Scientific Fund (Grant Nos. 81225010, 81327002 and 31170961), National 863 Hi-tech Project (Nos. 2012AA022703 and 2014AA020700), and Special Project for Nanotechnology from Shanghai (No. 13NM1401500). We would like to thank Instrumental Analysis Center of SJTU for the TEM analysis, dark-field imaging and ICP measurements.

\section{References}

[1] J. Ferlay, H.R. Shin, F. Bray, et al., Estimates of worldwide burden of cancer in 2008: GLOBOCAN 2008. Int. J. Cancer, 2010, 127: 2893-2917.

[2] R. Siegel, D. Naishadham, and A. Jemal, Cancer statistics, 2013. CA Cancer J Clin, 2013, 63: 11-30.

[3] T. Takahashi, Y. Saikawa, and Y. Kitagawa, Gastric cancer: current status of diagnosis and treatment. Cancers (Basel), 2013, 5: 48-63.

[4] I. Proserpio, S. Rausei, S. Barzaghi, et al., Multimodal treatment of gastric cancer. World Journal of Gastrointestinal Surgery, 2014, 6: 55-58.

[5] D. Zhang, D. Fan, New insights into the mechanisms of gastric cancer multidrug resistance and future perspectives. Future Oncol, 2010, 6: 527-537.

[6] D. Cui, L. Zhang, X.J. Yan, et al., A microarraybased gastric carcinoma prewarning system. World $J$ Gastroenterol, 2005, 11: 1273-1282.

[7] C. Bao, J. Conde, F. Pan, et al., Gold nanoprisms as a hybrid in vivo cancer theranostic platform for in situ photoacoustic imaging, angiography, and localized hyperthermia. Nano Research, 2016, 9: 1043-1056.

[8] M.F. Clarke, J.E. Dick, P.B. Dirks, et al., Cancer stem cells-perspectives on current status and future directions: Aacr workshop on cancer stem cells. Cancer Res, 2006, 66: 9339-9344.

[9] C. Zhang, C. Li, F. He, et al., Identification of cd44+cd24+ gastric cancer stem cells. J Cancer Res Clin Oncol, 2011, 137: 1679-1686.

[10] K. Wang, J. Ruan, Q. Qian, et al., BRCAA1 monoclonal antibody conjugated fluorescent magnetic nanoparticles for in vivo targeted magnetofluorescent imaging of gastric cancer. J Nanobiotechnol, 20111, 9: 23.

[11] C. Wang, C.C. Bao, S.J. Liang, et al., HAI-178 antibodyconjugated fluorescent magnetic nanoparticles for targeted imaging and simultaneous therapy of gastric cancer. Nanoscale Res. Lett, 2014, 9: 274.

[12] J. Ruan, H. Song, Q.R. Qian, et al., HER2 monoclonal antibody conjugated RNase-A-associated CdTe quantum dots for targeted imaging and therapy of gastric cancer. Biomaterials, 2012, 33: 7093-7102.

[13] M. He, P. Huang, C.L. Zhang, et al., Dual phasecontrolled synthesis of uniform lanthanide-doped $\mathrm{NaGdF}_{4}$ upconversion nanocrystals via an $\mathrm{OA} /$ ionic liquid two-phase system for in vivo dual-modality imaging. Advanced Functional Materials, 2011, 21: 4470-4477.

[14] Z.M. Li, P. Huang, X.J. Zhang, et al., RGD-conjugated dendrimer-modified gold nanorods for in vivo tumor targeting and photothermal therapy. Molecular Pharmaceutics, 2010, 7: 94-104.

[15] P. Huang, J. Lin, X.S. Wang, et al., Light-triggered theranostics based on photosensitizer-conjugated carbon dots for simultaneous enhanced-fluorescence imaging and photodynamic therapy. Adv. Mater, 2012, 24: 5104-5110.

[16] Z.J. Zhou, C.L. Zhang, Q.R. Qian, et al., Folic acidconjugated silica capped gold nanoclusters for targeted fluorescence/X-ray computed tomography imaging. Journal of Nanobiotechnology, 2013, 11: 17.

[17] C.L. Zhang, Z.J. Zhou, Q.R. Qian, et al., Glutathionecapped fluorescent gold nanoclusters for dual-modal fluorescence/X-ray computed tomography imaging. $J$. Mater. Chem. B, 2013, 1: 5045-5053.

[18] S.J. Liang, C. Li, C.L. Zhang, et al., CD44v6 Monoclonal Antibody-Conjugated Gold Nanostars for Targeted Photoacoustic Imaging and Plasmonic Photothermal Therapy of Gastric Cancer Stem-like Cells. Theranostics, 2015, 5: 970-984.

[19] D.X. Cui, C.L. Zhang, B. Liu, et al., Regression of gastric cancer by systemic injection of RNA nanoparticles carrying both ligand and siRNA. Sci Rep, 2015: 10726.

[20] P. Huang, C. Xu, J. Lin, et al., Folic acid-conjugated graphene oxide loaded with photosensitizers for targeting photodynamic therapy. Theranostics,. 2011, 1: 240-250.

[21] J.P. Zhang, Y. Song, C.L. Zhang, et al., Circulating MiR$16-5 p$ and MiR-19b-3p as two novel potential biomarkers to indicate progression of gastric cancer. Theranostics, 2015, 5, 733-745.

[22] Y.X. Zhang, G. Gao, H.J. Liu, et al.,.Identification of volatile biomarkers of gastric cancer cells and ultrasensitive electrochemical detection based on sensing interface of au-ag alloy coated MWCNTs. Theranostics, 2014, 4: 154-162.

[23] C. Wang, C.C. Bao, S.J. Liang, et al., RGD-conjugated silica-coated gold nanorods on the surface of carbon nanotubes for targeted photoacoustic imaging of gastric cancer. Nanoscale Res. Lett, 2014, 9: 264.

[24] P. Huang, L. Bao, C.L. Zhang, et al., Folic acidconjugated silica-modified gold nanorods for X-ray/CT imaging-guided dual-mode radiation and photo-thermal therapy. Biomaterials, 2011, 32: 9796-9809.

[25] Y. Liu, M. Yang, J. Zhang, et al., Human induced pluripotent stem cells for tumor targeted delivery of gold nanorods and enhanced photothermal therapy. ACS Nano, 2016, 10: 2375-2385.

[26] S.H. Chen, P. Huang, Z.H. Wang, et al., Self-assembly of gold nanoparticles to silver microspheres as highly efficient 3D SERS substrates. Nanoscale Res. Lett, 2013, 8: 168.

[27] X.S. Wang, D.P. Yang, P. Huang, et al., Hierarchically assembled $\mathrm{Au}$ microsphers and ser urchin-like architectures: formation mechanism and SERS study. Nanoscale, 2012, 4: 7766. 
[28] L. Wang, T. Guo, Q. Lu, et al., Sea-urchin-like Au nanocluster with surface-enhanced Raman scattering in detecting epidermal growth factor receptor (EGFR) mutation status of malignant pleural effusion. ACS Applied Materials \& Interface, 2015, 7: 359-369.

[29] S.H. Chen, D.B. Liu, Z.H. Wang, et al., Picomolar detection of mercuric ions by means of gold silver coreshell nanorod. Nanoscale, 2013, 5: 6731-6735

[30] C.C. Bao, N. Beziere, P. del Pino, et al., Gold nanoprisms as optoacoustic signal nanoamplifiers for in vivo bioimaging of gastrointestinal cancers. Small, 2013, 9: 68-74.

[31] C.L. Zhang, C. Li, Y.L. Liu, et al., Gold nanoclustersbased nanoprobes for simultaneous fluorescence imaging and targeted photodynamic therapy with superior penetration and retention behavior in tumors. Advanced Fun. Mater, 2015, 25: 1314-1325.

[32] C. Bao, J. Conde, E. Polo, et al., A promising road with challenges: where gold nanoparticles are in translational research? Nanomedicine, 2014, 9: 2353-2370.

[33] C.J. Orendorff, T.K. Sau, and C.J. Murphy, Shapedependent plasmon-resonant gold nanoparticles. Small, 2006, 2: 636-639.

[34] X. Huang, I.H. El-Sayed, W. Qian, et al., Cancer cell imaging and photothermal therapy in the near-infrared region by using gold nanorods. Journal of the American Chemical Society, 2006, 128: 2115-2120.

[35] L.L. Feng, G. Gao, P. Huang, et al., Optical proerties and catalytic activity of bimetallic gold -silver nanoparticles. Nano Biomed Eng, 2010, 2: 258-267.

[36] C.J. Orendorff, T.K. Sau, C.J. Murphy, Shape-dependent plasmon-resonant gold nanoparticles. Small, 2006, 2(5): 636-639.

[37] B. Pelaz, P. del Pino, Synthesis and applications of gold nanoparticles. Frontiers of Nanoscience, 2012, 4: 3-33.

[38] J. Conde, C.C. Bao, D.X. Cui, et al., Antibody-drug gold nanoantennas with Raman spectroscopic fingerprints for in vivo tumor theranostics J. Controlled Release, 2014, 183: 87-93.

[39] C.C. Bao, J. Conde, J. Curtin, et al., Bioresponsive antisense DNA gold nanobeacons as a hybrid in vivo theranostics platform for the inhibition of cancer cells and metastasis. Sci Rep, 2015, 5: 12197.

[40] B.F. Pan, D.X. Cui, C. Ozkan, et al., DNA-templated ordered array of gold nanorods in one and two dimesnions. J. Phys. Chem. C, 2007, 111 (34): 1257212576.

[41] G.V. Hartland, G. Schatz, Virtual issue: plasmon resonances-a physical chemistry perspective. The Journal of Physical Chemistry C, 2011, 115(31): 15121-15123.

[42] S.M. Leto, F. Sassi, I. Catalano, et al., Sustained inhibition of HER3 and EGFR is necessary to induce regression of HER2-amplified gastrointestinal carcinomas. Clin Cancer Res, 2015, 21(24): 5519-5531.

[43] J. Han, J. Zhang, M. Yang, et al., Glucose-functionalized Au nanoprisms for optoacoustic imaging and nearinfrared photothermal therapy. Nanoscale, 2016, 8: 492499.

[44] C. Li, S. Liang, C. Zhang, et al., Allogenic dendritic cell and tumor cell fused vaccine for targeted imaging and enhanced immunotherapeutic efficacy of gastric cancer. Biomaterials, 2015, 54: 177-187.

[45] J. Conde, C.C. Bao, Y.Q. Tan,et al., Dual targeted immunotherapy via in vivo delivery of biohybrid RNAipeptide nanoparticles to tumor-associated macrophages and cancer cells. Adv. Fun. Mater, 2015, 25: 4183-4194.

[46] W. Lu, Q. Huang, G. Ku,et al., Photoacoustic imaging of living mouse brain vasculature using hollow gold nanospheres. Biomaterials, 2010, 31(9): 2617-2626.

[47] M.A. Mackey, M.R. Ali, L.A. Austin, et al., The most effective gold nanorod size for plasmonic photothermal therapy: theory and in vitro experiments. The Journal of Physical Chemistry B, 2014, 118(5): 1319-1326.

[48] Y. Li, T. Wen, R. Zhao, et al., Localized electric field of plasmonic nanoplatform enhanced photodynamic tumor therapy. ACS Nano, 2014, 8(11): 11529-11542.

[49] A.M. Alkilany, L.B. Thompson, S.P. Boulos, et al., Gold nanorods: their potential for photothermal therapeutics and drug delivery, tempered by the complexity of their biological interactions. Advanced Drug Delivery Reviews, 2012, 64(2): 190-199.

[50] N.J. Durr, T. Larson, D.K. Smith, et al., Two-photon luminescence imaging of cancer cells using molecularly targeted gold nanorods. Nano Letters, 2007, 7(4): 941945.

Copyright $@ 2016$ Shouhui Chen, Chenchen Bao, Chunlei Zhang, Yao Yang, Kan Wang, Bhaskara V. Chikkaveeraiah, Zhihua Wang, Xinglu Huang, Fei Pan, Kun Wang, Xiao Zhi, Jian Ni, Jesus M. de la Fuente, and Jie Tian. This is an openaccess article distributed under the terms of the Creative Commons Attribution License, which permits unrestricted use, distribution, and reproduction in any medium, provided the original author and source are credited. 